\title{
Ovarian cancer population screening and mortality after long-term follow-up in the UK Collaborative Trial of Ovarian Cancer Screening (UKCTOCS): a randomised controlled trial
}

\author{
Usha Menon, Aleksandra Gentry-Maharaj, Matthew Burnell, Naveena Singh, Andy Ryan, Chloe Karpinskyj, Giulia Carlino, Julie Taylor, \\ Susan K Massingham, Maria Raikou, Jatinderpal K Kalsi, Robert Woolas, Ranjit Manchanda, Rupali Arora, Laura Casey, Anne Dawnay, Stephen Dobbs, \\ Simon Leeson, Tim Mould, Mourad W Seif, Aarti Sharma, Karin Williamson, Yiling Liu, Lesley Fallowfield, AlistairJ McGuire, Stuart Campbell, \\ Steven J Skates, IanJJacobs, Mahesh Parmar
}

\section{Summary}

Background Ovarian cancer continues to have a poor prognosis with the majority of women diagnosed with advanced disease. Therefore, we undertook the UK Collaborative Trial of Ovarian Cancer Screening (UKCTOCS) to determine if population screening can reduce deaths due to the disease. We report on ovarian cancer mortality after long-term follow-up in UKCTOCS.

Methods In this randomised controlled trial, postmenopausal women aged 50-74 years were recruited from 13 centres in National Health Service trusts in England, Wales, and Northern Ireland. Exclusion criteria were bilateral oophorectomy, previous ovarian or active non-ovarian malignancy, or increased familial ovarian cancer risk. The trial management system confirmed eligibility and randomly allocated participants in blocks of 32 using computer generated random numbers to annual multimodal screening (MMS), annual transvaginal ultrasound screening (USS), or no screening, in a 1:1:2 ratio. Follow-up was through national registries. The primary outcome was death due to ovarian or tubal cancer (WHO 2014 criteria) by June 30, 2020. Analyses were by intention to screen, comparing MMS and USS separately with no screening using the versatile test. Investigators and participants were aware of screening type, whereas the outcomes review committee were masked to randomisation group. This study is registered with ISRCTN, 22488978, and ClinicalTrials.gov, NCT00058032.

Findings Between April 17, 2001, and Sept 29, 2005, of 1243282 women invited, 202638 were recruited and randomly assigned, and 202562 were included in the analysis: 50625 (25.0\%) in the MMS group, $50623(25.0 \%)$ in the USS group, and $101314(50 \cdot 0 \%)$ in the no screening group. At a median follow-up of $16 \cdot 3$ years (IQR 15·1-17.3), 2055 women were diagnosed with tubal or ovarian cancer: $522(1.0 \%)$ of 50625 in the MMS group, $517(1.0 \%)$ of 50623 in the USS group, and $1016(1.0 \%)$ of 101314 in the no screening group. Compared with no screening, there was a $47 \cdot 2 \%(95 \% \mathrm{CI}$ 19.7 to $81 \cdot 1)$ increase in stage I and $24.5 \%(-41.8$ to -2.0$)$ decrease in stage IV disease incidence in the MMS group. Overall the incidence of stage I or II disease was $39 \cdot 2 \%(95 \%$ CI $16 \cdot 1$ to $66 \cdot 9)$ higher in the MMS group than in the no screening group, whereas the incidence of stage III or IV disease was $10 \cdot 2 \%(-21 \cdot 3$ to $2 \cdot 4)$ lower. 1206 women died of the disease: $296(0.6 \%)$ of 50625 in the MMS group, 291 (0.6\%) of 50623 in the USS group, and $619(0 \cdot 6 \%)$ of 101314 in the no screening group. No significant reduction in ovarian and tubal cancer deaths was observed in the MMS $(\mathrm{p}=0 \cdot 58)$ or USS $(\mathrm{p}=0 \cdot 36)$ groups compared with the no screening group.

Interpretation The reduction in stage III or IV disease incidence in the MMS group was not sufficient to translate into lives saved, illustrating the importance of specifying cancer mortality as the primary outcome in screening trials Given that screening did not significantly reduce ovarian and tubal cancer deaths, general population screening cannot be recommended.

Funding National Institute for Health Research, Cancer Research UK, and The Eve Appeal.

Copyright (C) 2021 The Author(s). Published by Elsevier Ltd. This is an Open Access article under the CC BY 4.0 license.

\section{Introduction}

Ovarian cancer remains the most deadly of all gynaecological cancers. Most patients (58\%) are diagnosed at an advanced stage (III or IV), which is associated with poor survival (5-year survival is $27 \%$ for stage III and $13 \%$ for stage IV ovarian cancer). ${ }^{1}$ The greater than $90 \%$ survival rates in women detected at stage $\mathrm{I}^{1}$ has spurred international efforts in early detection, spanning across four decades..$^{2-6}$ All trials have used combinations of the biomarker CA125 and pelvic imaging using transvaginal ultrasound scans (TVS). Despite these extensive endeavours, to date there is no evidence that screening for ovarian cancer saves lives. ${ }^{7-9}$

In our multicentre randomised trial (UK Collaborative Trial of Ovarian Cancer Screening [UKCTOCS]), 202638 women from the general population were

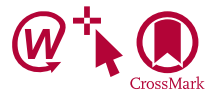

Published Onlin May 12, 2021 https://doi.org/10.1016/ S0140-6736(21)00731-5 See Online/Comment https://doi.org/10.1016/ S0140-6736(21)01061-8

MRC Clinical Trials Unit, Institute of Clinical Trials and Methodology (Prof U Menon FRCOG, A Gentry-Maharaj PhD, M Burnell PhD,

A Ryan PhD, C Karpinskyj MSc, G Carlino MSc,

SK Massingham RGN,

Prof M Parmar DPhil), Clinical Epidemiology, Institute of Health Informatics

(JTaylor MSc), and Department of Women's Cancer, Institute

for Women's Health

(J K Kalsi PhD,

Prof IJ Jacobs FRCOG)

University College London,

London, UK; Department of

Cellular Pathology

(Prof N Singh FRCPath,

L Casey FRCPath), Department

of Clinical Biochemistry

(A Dawnay PhD), and

Department of Gynaecologica Oncology

(Prof R Manchanda PhD), Barts Health NHS Trust, London, UK Department of Health Policy, London School of Economics and Political Science, London, UK (M Raikou PhD, Prof A J McGuire PhD); Department of Economics, University of Piraeus, Athens, Greece (M Raikou); Department of Gynaecological Oncology Queen Alexandra Hospital, Portsmouth, UK (R Woolas FRCOG); Wolfson Institute of Preventive Medicine, CRUK Barts Cancer Centre, Queen Mary University of London, London, UK (Prof R Manchanda PhD); Department of Cellular Pathology (R Arora FRCPath) and Department of 
Gynaecological Oncology (T Mould FRCOG), University College London Hospitals NHS Trust, London, UK; Department of Gynaecological Oncology, Belfast City Hospital, Belfast, UK (S Dobbs FRCOG); Department of Obstetrics and Gynaecology, Ysbyty Gwynedd, Bangor, UK (S Leeson FRCOG); Division of Gynaecology and Cancer Sciences, St Mary's Hospital, Manchester, UK (M W Seif FRCOG); Department of Obstetrics and Gynaecology, University Hospital of Wales, Cardiff, UK (A Sharma FRCOG); Department of Gynaecological Oncology, Nottingham City Hospital, Nottingham, UK (K Williamson FRCOG); MGH Biostatistics, Department of Medicine, Massachusetts General Hospital, Boston, MA USA (S J Skates PhD, Y Liu MS); Department of Medicine, Harvard Medical School, Boston, MA, USA (S J Skates); Sussex Health Outcomes Research and Education in Cancer, SHORE-C, Brighton and Sussex Medical School, University of Sussex, Brighton, UK (Prof L Fallowfield DPhil); Create Health, London, UK (Prof S Campbell FRCOG); Department of Women's Health, University of

New South Wales, Sydney, NSW, Australia (Prof IJ Jacobs) Correspondence to: Prof Usha Menon, MRC Clinical Trials Unit, Institute of Clinical

Trials and Methodology, University College London, London WC1V 6LJ, UK u.menon@ucl.ac.uk

\section{Research in context}

\section{Evidence before this study}

We searched PubMed from Jan 1, 2015, to Dec 31, 2020, with no language restrictions for randomised controlled trials for ovarian cancer screening that reported mortality data. The following keywords were used to search the database: "ovarian cancer" AND "randomised controlled trial" AND "screening" AND "mortality". We found two relevant publications. In the UK Collaborative Trial of Ovarian Cancer Screening (UKCTOCS; $\mathrm{n}=202638$ ), at a median follow-up of 11.1 years, no significant reduction in deaths from ovarian cancer was seen in either of the screen groups (multimodal or ultrasound) compared with the no screening group. A reduction in deaths was seen but was delayed and only apparent after about 7 years. There was a suggestion that $15 \%$ fewer women in the multimodal screening group and $11 \%$ fewer in the ultrasound screening group died from ovarian cancer compared with the no screening group. Additionally, a significantly greater proportion (13\%) of women with ovarian cancers in the multimodal group but not in the ultrasound group were found at an earlier stage (stage I and II) compared with the no screening group. As the data did not definitively answer the question of whether screening saved lives, follow-up was continued to gather more evidence. The Ovarian Cancer Screening arm of the Prostate Lung Colorectal Ovarian (PLCO) cancer trial in the USA is the only other large randomised controlled trial $(n=78216)$ to explore mortality benefit. Following extended follow-up (median

randomly assigned to two annual screening groupsmultimodal screening (MMS; longitudinal CA125 and second line TVS) and ultrasound screening (USS; TVS first and second-line test), and a no screening group. We reported previously (median follow-up of $11 \cdot 1$ years), that an absolute proportion of $13 \%$ more women with ovarian, tubal, and peritoneal cancer were diagnosed with stage I or II disease in the MMS group than in the no screening group. There was no change in stage in the USS group. There was no evidence of a reduction in disease-specific deaths in either screened group compared with the no screening group using the Cox version of the log-rank test. The observed reduction in deaths was delayed and the cumulative mortality curves appeared to be diverging at the time of previous reporting. ${ }^{9}$ Therefore, we aimed to continue follow-up and report here on the longterm mortality effects of ovarian cancer screening in UKCTOCS

\section{Methods}

For the protocol see http:// ukctocs.mrcctu.ucl.ac.uk/ media/1066/ukctocs-protocol_ v90_19feb2020.pdf

\section{Study design and participants}

We did a randomised controlled trial (UKCTOCS) of postmenopausal women aged 50-74 years from the general population recruited through 13 centres in National Health Service (NHS) Trusts in England, Wales, and Northern Ireland with use of age-sex registers of 27 primary care trusts. ${ }^{10}$
14.7 years), the trial confirmed previous findings of no ovarian cancer mortality reduction between the screen and control arms.

\section{Added value of this study}

Long-term follow-up (median follow-up $>16$ years after recruitment) in the largest ovarian cancer screening trial, to our knowledge, provides definitive new evidence that neither screening approaches used in UKCTOCS reduced deaths from ovarian cancer, compared with no screening. This result was despite a $47 \cdot 2 \%$ increase in incidence of women with ovarian and tubal cancer diagnosed at stage I and $24.5 \%$ decrease in those diagnosed with stage IV disease in the multimodal group compared with the no screening group. Importantly, however, there was only a $10 \cdot 2 \%$ decrease in overall incidence of stage III or IV disease.

\section{Implications of all the available evidence}

General population screening for ovarian and tubal cancer with either approach used in UKCTOCS cannot currently be recommended. We need a screening strategy that can detect ovarian and tubal cancer in asymptomatic women even earlier in its course and in a larger proportion of women than the tests used in the trial. Meanwhile, our results emphasise the importance of having ovarian and tubal cancer mortality as the primary outcome in screening trials.

We commissioned specialised software from the NHS to randomly select women aged 50-74 years and then flag them on primary care trusts' registers and allow electronic transfer of their personal and general practice details. We then sent women personal invitations and logged replies on the trial management system. Women attended a recruitment clinic at the regional centre where they viewed an information video, completed a recruitment questionnaire, and provided written consent and a baseline serum sample. We scanned recruitment questionnaires at the coordinating centre into a bespoke trial management system.

Inclusion criteria were 50-74 years of age and postmenopausal status. Exclusion criteria were bilateral oophorectomy, previous ovarian or active non-ovarian malignancy, or increased familial ovarian cancer risk.

Ethical approval was provided by the UK North West Multicentre Research Ethics Committees (00/8/34) on June 23, 2000. All participants provided written informed consent. The trial design has been previously published and the protocol is available online. ${ }^{9-12}$

\section{Randomisation and masking}

The trial management system confirmed eligibility and then randomly allocated women using the Visual Basic randomisation statement and the RND function to annual screening using the MMS or USS strategy, or no 
screening in a 1:1:2 ratio. The trial management system allocated a set of 32 random numbers to each regional centre, of which eight were allocated to MMS, eight to USS, and the remaining 16 to no screening. We randomly allocated each successive volunteer within the regional centre to one of the numbers and subsequently randomly allocated them into a group. Investigators and participants were aware of group allocation but members of the outcomes committee were masked to randomisation group.

\section{Procedures}

Annual screening in the MMS group used serum CA125 measurements, with the pattern over time interpreted using the risk of ovarian cancer (ROCA) calculation, ${ }^{13}$ which identifies significant rises in CA125 concentration above baseline. On the basis of risk, women were triaged to normal (annual screening), intermediate (repeat CA125 ROCA test in 3 months), and elevated (repeat CA125 ROCA test and transvaginal USS as a second-line test in 6 weeks) risk. Annual screening in the USS group used TVS as the primary test, which was classified as normal (annual screening), unsatisfactory (repeat in 3 months), or abnormal (scan with a senior ultrasonographer within 6 weeks). In both groups, women with persistent abnormalities were assessed by a trial clinician and referred to the NHS where they underwent further investigation or surgery. We deemed women who had surgery or a biopsy for suspected ovarian cancer after clinical assessment as screen positive.

Women were linked using their NHS number to national cancer and death registration data and Hospital Episode Statistics records (appendix p 4). An additional questionnaire was sent in June, 2020, to a subset of participants who had either exited the national registries or for whom it was not possible from Hospital Episode Statistics data to ascertain if both ovaries had been removed.

Throughout the trial, we interrogated the available sources to identify women diagnosed with any of 19 International Classification of Diseases (ICD)-10 codes for possible ovarian or tubal cancer and retrieved copies of medical notes. ${ }^{12,14}$ The only exception was women with malignant neoplasm without specification of site (ICD-10 C80), who also had another non-ovarian, tubal, or peritoneal cancer registration. Medical notes, with any mention of randomisation group redacted, were reviewed by the outcomes review committee consisting of gynaecological pathologists and oncologists (NS, RM, RW, RA, LC, AS, and KW). The outcomes review committee assigned cancer site (ie, whether ovarian, tubal, or other site using a previously audited prespecified algorithm),$^{14}$ Federation of Gynecology and Obstetrics (FIGO) 2014 stage, grade, morphology, ovarian cancer type, and cause of death. We defined ovarian and tubal cancer using the WHO 2014 classification $^{15,16}$ and death due to ovarian and tubal cancer based on disease progression (new or increases in size of previously documented lesions on imaging, clinical worsening, or rising biomarker concentrations). In the WHO 2014 classification, the definition for primary peritoneal cancers was revised. The outcomes review committee chair (NS) reviewed all 41 cancers previously classified as primary peritoneal as per WHO 2003 classification. ${ }^{9}$ The outcomes review committee re-staged all ovarian and tubal cancers using FIGO 2014 criteria (previously staged using FIGO 2003) diagnosed in 2001-14.

\section{Outcomes}

The primary outcome was death due to ovarian (ICD-10 C56) or tubal (ICD-10 C57.0) cancer. Ovarian cancer includes primary non-epithelial ovarian cancer, borderline epithelial ovarian cancer, and invasive epithelial ovarian cancer. As stated above, ovarian cancer was defined using the revised WHO 2014 definition..$^{15,16}$ The site assignment is in contrast to the previous mortality analysis (censorship Dec 31, 2014), which used the WHO 2003 definition. ${ }^{17}$ The majority (40 of 41) of previously classified primary peritoneal cancers using WHO 2003 criteria were reclassified as ovarian or tubal cancers. Secondary outcomes were ovarian and tubal cancer incidence and stage. For all outcomes, subgroup analysis was undertaken for invasive epithelial ovarian and tubal cancer. All outcome data were kept confidential until unmasking. Case fatality rate by stage was a posthoc outcome.

\section{Statistical analysis}

At previous analysis (censorship Dec 31, 2014), there were 358 ovarian and tubal cancer deaths in the no screening group. ${ }^{9}$ Compared with the no screening group, the mean estimated relative mortality reduction in deaths was $11 \%$ (Cox model $\mathrm{p}=0 \cdot 24$ ) in the MMS group and $9 \%$ (Cox model $\mathrm{p}=0 \cdot 32$ ) in the USS group. Any mortality reduction was only apparent about 7 years after randomisation. 162 (45\%) of 358 of the deaths in the no screening group during 2001-14 occurred before 7 years. In 2015, for the no screening versus MMS or USS comparisons, we estimated that an additional 233 no screening group events would give $80 \%$ power at a two-sided $5 \%$ significance level for a difference in relative mortality of $25 \%$ during long-term (2015-20) follow-up, conditional on the observed mortality reduction of $11 \%$. This estimate translated to a target sample size of 591 overall events in the no screening group: all 233 new and $73 \%$ (431 of 591) of total no screening group events would occur beyond 7 years. No formal adjustment was made to the test for having previously analysed the data in 2015 or making two screen group comparisons. Instead, we decided to openly describe the multiplicity issues and acknowledge the unadjusted $\mathrm{p}$ values. As the number of events were less than anticipated on the planned censorship date 
of Dec 31, 2018, follow-up was extended with a new censorship date of June 30, 2020.

Descriptive statistics regarding ovarian cancer death and incidence were created including tabulations of histology, stage, and screen type by group. The primary mortality analysis was changed from the 2016 report, in which we used a Cox version of the log-rank test, ${ }^{9}$ which is most powerful under proportional hazards. For the current analysis, we extensively discussed the best approach within trial management and trial steering committees, and consulted 12 independent international statistical, trial, and screening experts. The details and rationale underpinning this important change are reported separately. ${ }^{18}$ In short, given the accumulating external evidence of delayed mortality effects in screening trials, most experts supported the change in primary analysis to a test that was sensitive to delayed effects. We chose the versatile test that was agnostic to the specific form of the screening effect. The versatile test, described in 2016, ${ }^{18}$ is a combination test of three log-rank test statistics $\left(Z_{1}, Z_{2}, Z_{3}\right)$, covering early, constant, and late effects respectively (appendix p 3).

All analyses were by intention to screen. The primary mortality analysis was an MMS versus no screening and

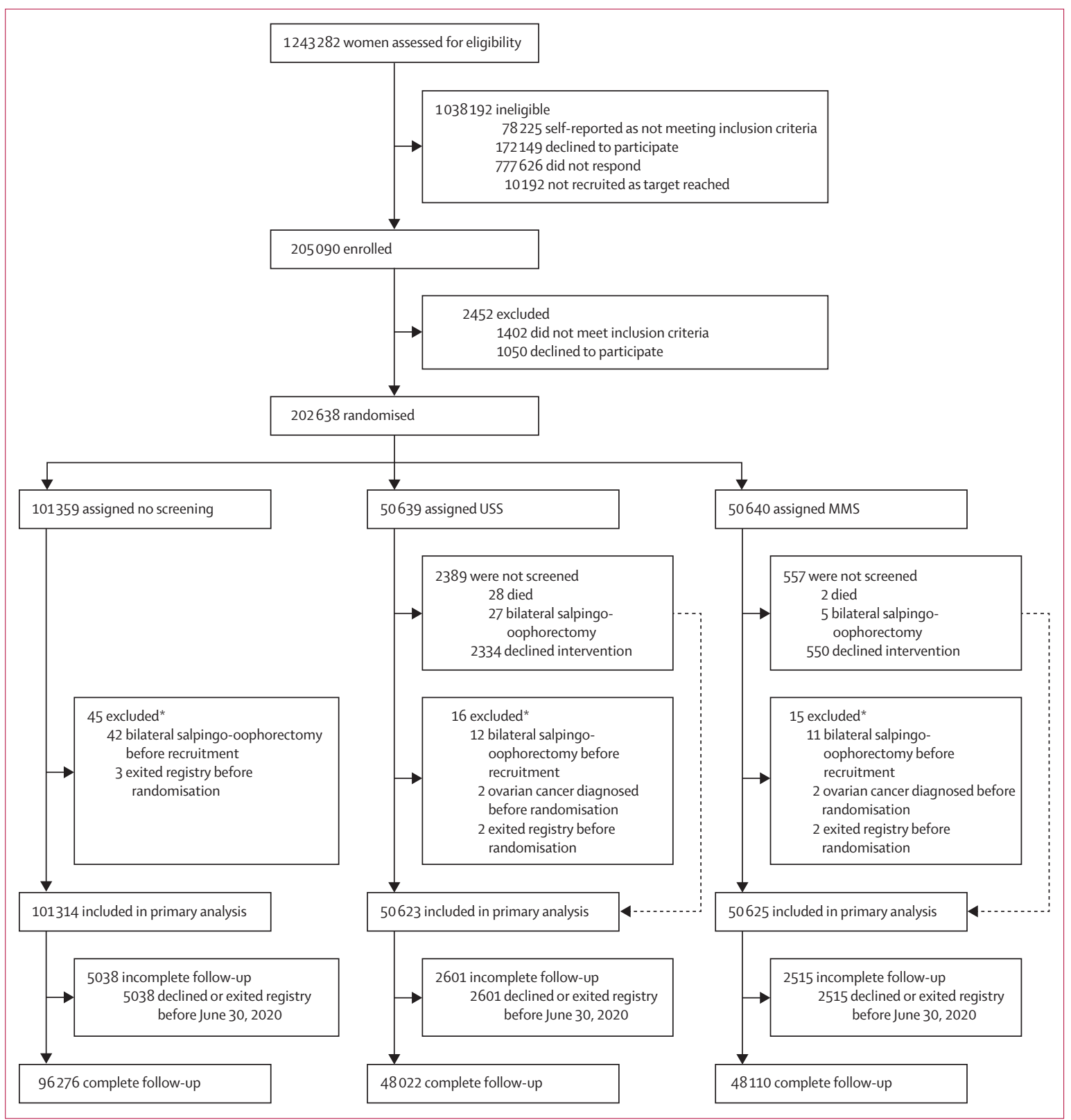

Figure 1: Trial profile

MMS=multimodal screening. USS=ultrasound screening. *Events occurred before recruitment, but were discovered after randomisation. 
USS versus no screening analysis of the primary outcome using the versatile test, ${ }^{19}$ with a Royston-Parmar model to estimate survival differences. We defined survival time from date of randomisation $\left(t_{0}=0\right)$ to date of death due to ovarian or tubal cancer or censorship, or sooner if the volunteer died of another cause or was lost to follow-up. No allowances were made for screening non-compliance (study groups) or contamination (no screening group). We describe potential time-dependent features of the screening effect by estimating the hazard ratio (HR) and the absolute survival difference at the prespecified timepoints of 5, 10, 15, and 18 years (maximal follow-up was 19.3 years) using a flexible parametric RoystonParmar model (appendix p 3).

We undertook two secondary analyses of the primary outcome. We fitted a proportional hazards Cox model to the primary outcome data. To allow for a formal analysis of the late effects of ovarian cancer, not subject to issues of data re-use and multiple testing, we also fitted a Cox model to the new data acquired since Jan 1, 2015. Both the methods and results of sensitivity analyses are detailed in the appendix (pp 8-9). Survival from diagnosis in women with ovarian and tubal cancer in the no screening group was also compared to national age and period adjusted survival rates at 1,5 , and 10 years. We undertook a subgroup analysis using the versatile test of invasive epithelial ovarian and tubal cancer death, in which other ovarian cancers were censored at death.

For the secondary outcome, cumulative incidence of ovarian and tubal cancer were presented graphically using standard Kaplan-Meier methods, based on time from randomisation to diagnosis. Death from other causes and bilateral salpingo-oophorectomy were censoring events. Administrative censorship was the same as for the mortality analysis (June 30, 2020). Ovarian and tubal cancer incidence rates were explored parametrically using a Royston-Parmar model that specifically allowed exploration of the underlying hazard functions for cancer incidence. For the secondary outcome of ovarian and tubal cancer incidence by stage, and the subgroup analysis of invasive epithelial ovarian and tubal cancers, we used incidence rate ratios with 95\% CIs to compare no screening versus MMS and USS groups separately. We also calculated stage-specific ovarian and tubal cancer case fatality rates.

We used Stata, version 16 (versatile test verswlr function) for all statistical analyses. Results were independently verified by a different statistician using $R$, version 4.0.2 (versatile test logrank.maxtest of $\mathrm{nph}$ package). This trial is registered with ISRCTN, 22488978, and ClinicalTrials.gov, NCT00058032.

\section{Role of the funding source}

The funders of the study had no role in study design, data collection, data analysis, data interpretation, or writing of the report.

\section{Results}

Between April 17, 2001, and Sept 29, 2005, we invited 1243282 women to participate and randomly assigned

\begin{tabular}{|c|c|c|c|c|c|c|}
\hline & \multirow[t]{2}{*}{ Total } & \multirow{2}{*}{$\begin{array}{l}\text { Screen } \\
\text { positives }\end{array}$} & \multicolumn{4}{|c|}{ Cancers not detected by screening } \\
\hline & & & $\begin{array}{l}\text { Screen negatives } \\
\leq 1 \text { year from last test } \\
\text { of screening episode }\end{array}$ & $\begin{array}{l}\text { Screen negatives } \\
>1 \text { year after last test } \\
\text { of screening episode }\end{array}$ & $\begin{array}{l}\text { Never attended } \\
\text { screening }\end{array}$ & $\begin{array}{l}\text { Diagnosed >1 year } \\
\text { after end of } \\
\text { screening }^{*}\end{array}$ \\
\hline \multicolumn{7}{|c|}{ Multimodal screening ( 50625 women, 789129 women-years) } \\
\hline Ovarian and tubal cancer & $522(100 \%)$ & $212(41 \%)$ & $41(8 \%)$ & $41(8 \%)$ & $3(1 \%)$ & $225(43 \%)$ \\
\hline Non-epithelial ovarian cancer & $16(100 \%)$ & $7(44 \%)$ & $2(13 \%)$ & $2(13 \%)$ & 0 & $5(31 \%)$ \\
\hline Borderline epithelial ovarian cancer & $54(100 \%)$ & $24(44 \%)$ & $10(19 \%)$ & $5(9 \%)$ & 0 & $15(28 \%)$ \\
\hline $\begin{array}{l}\text { Invasive epithelial ovarian and tubal } \\
\text { cancer }\end{array}$ & $452(100 \%)$ & $181(40 \%)$ & $29(6 \%)$ & $34(8 \%)$ & $3(1 \%)$ & $205(45 \%)$ \\
\hline \multicolumn{7}{|c|}{ Ultrasound screening (50 623 women, 790231 women-years) } \\
\hline Ovarian and tubal cancer & $517(100 \%)$ & $164(32 \%)$ & $63(12 \%)$ & $50(10 \%)$ & $19(4 \%)$ & $221(43 \%)$ \\
\hline Non-epithelial ovarian cancer & $13(100 \%)$ & $11(85 \%)$ & 0 & $1(8 \%)$ & 0 & $1(8 \%)$ \\
\hline Borderline epithelial ovarian cancer & $59(100 \%)$ & $48(81 \%)$ & $2(3 \%)$ & $1(2 \%)$ & $3(5 \%)$ & $5(8 \%)$ \\
\hline $\begin{array}{l}\text { Invasive epithelial ovarian and tubal } \\
\text { cancer }\end{array}$ & $445(100 \%)$ & $105(24 \%)$ & $61(14 \%)$ & $48(11 \%)$ & $16(4 \%)$ & $215(48 \%)$ \\
\hline \multicolumn{7}{|c|}{ No screening (101314 women, 1577517 women-years) } \\
\hline Ovarian and tubal cancer & $1016+(100 \%)$ & .. & .. & $514(51 \%)$ & .. & $499(49 \%)$ \\
\hline Non-epithelial ovarian cancer & $17(100 \%)$ & .. & .. & $7(41 \%)$ & .. & $10(59 \%)$ \\
\hline Borderline epithelial ovarian cancer & $91(100 \%)$ &. & .. & $50(55 \%)$ &. & $41(45 \%)$ \\
\hline $\begin{array}{l}\text { Invasive epithelial ovarian and tubal } \\
\text { cancer }\end{array}$ & $905(100 \%)$ & .. & .. & $457(50 \%)$ & .. & $448(50 \%)$ \\
\hline
\end{tabular}


$202638(16 \cdot 3 \%$ of 1243282 ; figure 1$) .{ }^{10}$ We followed-up participants until June 30, 2020. The final cohort eligible for analysis consisted of 202562 (>99.9\%) of 202638 women: 50625 (>99.9\%) in the MMS group, 50623 (>99.9\%) in the USS group, and 101314 (>99.9\%) in the no screening group. We excluded $76(<0 \cdot 1 \%)$ women $(15[<0 \cdot 1 \%]$ in the MMS group; $16[<0 \cdot 1 \%]$ in the USS group; and $45[<0 \cdot 1 \%]$ in the no screening group; figure 1) as they had bilateral salpingo-oophorectomy, ovarian cancer before joining the trial, or had exited the registry before randomisation. As previously reported, baseline characteristics were balanced between the groups. ${ }^{9}$ Screening ended on Dec 31, 2011. We undertook
673345 annual screens: 345570 in the MMS group and 327775 in the USS group. Compliance with screening was high $(81 \%$ in the MMS group and $78 \%$ in the USS group) with women undergoing a median of eight annual screens. ${ }^{?}$

After the end of annual screening on Dec 31, 2011, all women were followed up until the censorship date of June 30, 2020. Complete follow-up until censorship or death was possible in 192478 (95.0\%) women (48110 [95.0\%] in the MMS group; 48022 [94.9\%] in the USS group; and 96276 [95.0\%] in the no screening group) resulting in 3.16 million women-years. Median follow-up was $16 \cdot 3$ years (IQR 15·1-17·3) for all groups.

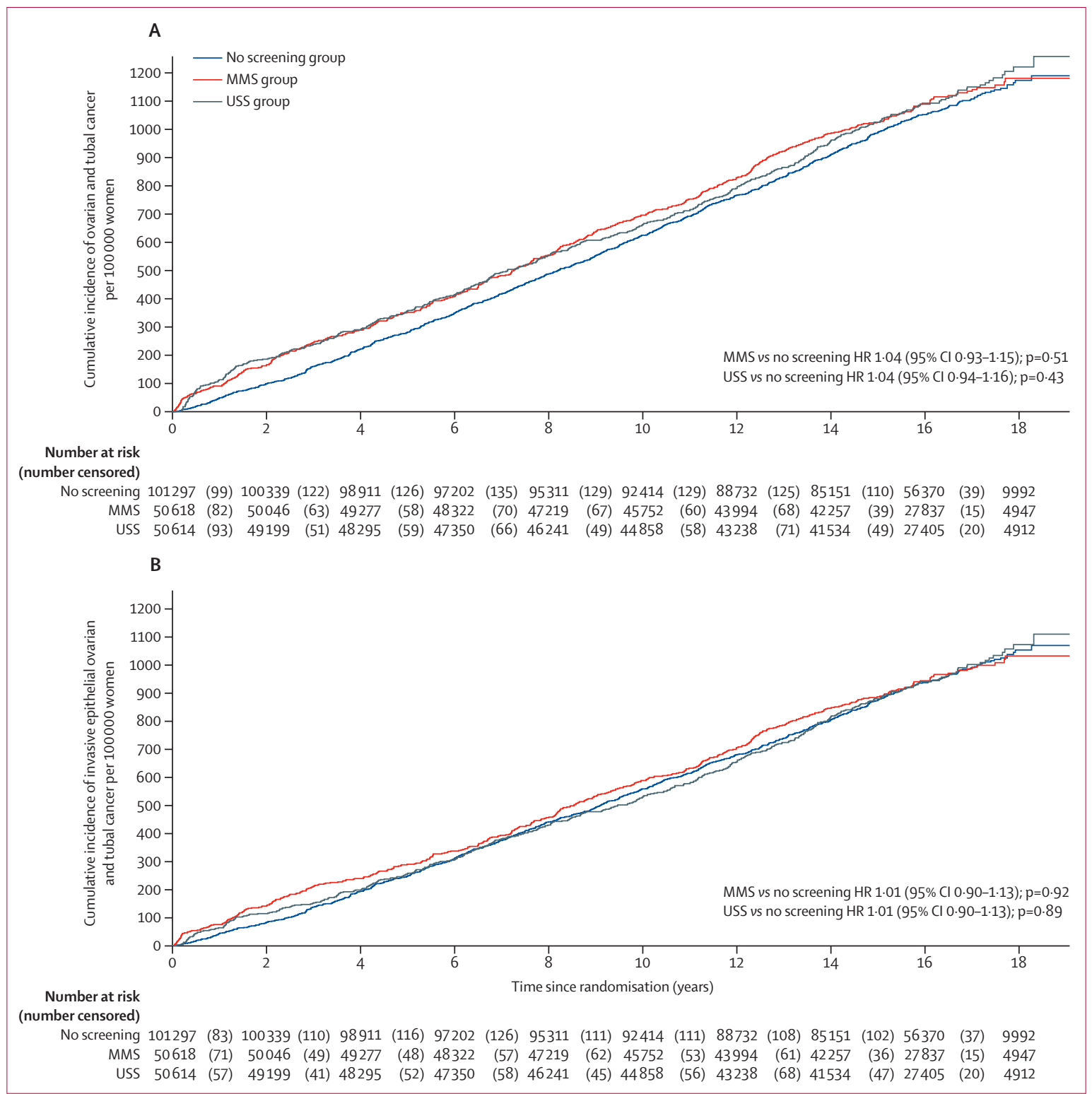

Figure 2: Kaplan-Meier cumulative incidence per 100000 women for all ovarian and tubal cancers (A) and for invasive epithelial ovarian and tubal cancers (B) $\mathrm{MMS}=$ multimodal screening. USS=ultrasound screening. $\mathrm{HR}=$ hazard ratio. 
We identified 4482 women with the 19 prespecified ICD-10 codes for possible ovarian and tubal cancer who were reviewed by the outcomes review committee (appendix p 5). Of them, 2055 (45.9\%) were confirmed to have ovarian or tubal cancer (table 1). The incidence of ovarian and tubal cancer per 100000 women-years was 67.7 (95\% CI 61 -9-73 · 5; 522 cancers; 770967 womenyears) in the MMS group, $68 \cdot 2$ (62.4-74.1; 517 cancers; 755677 women-years) in the USS group, and 65.4 (61.4-69.4; 1016 cancers; 1552703 women-years) in the no screening group (appendix p 11). Figure 2A provides Kaplan-Meier cumulative cancer rates for all women and figure 2B provides Kaplan-Meier cumulative cancer rates for invasive epithelial ovarian and tubal cancers. Both plots show a greater number of cancer diagnoses in the screening groups during the first screening year, reflecting the lead time to diagnosis achieved by screening. The difference was largely maintained throughout the screening phase before apparent catch-up by the no screening group during the extended period of follow-up after the end of screening. However, the pattern of catch- up in the USS group was less pronounced, and this observation is elucidated by the Royston-Parmar model hazard functions (appendix pp 19-20), in which the rate of cancer incidence drops below the no screening group between years 4-14 approximately, before rising back above the no screening group rate.

Overall, $1805(87 \cdot 8 \%)$ of 2055 women (452 [0.9\%] in the MMS group; 445 [0.9\%] in the USS group; 905 [0.9\%] in the no screening group) had invasive epithelial ovarian or tubal cancers. The proportion of the most aggressive type II cancers $(79.2 \%$ in the MMS group; $82 \cdot 2 \%$ in the USS group; and $76.4 \%$ in the no screening group) was similar across the groups (appendix p 6). At 9.5 years after the end of screening, compared with the no screening group, there was a $47 \cdot 2 \%$ (95\% CI 19.7 to $81 \cdot 1)$ higher incidence of stage I disease and a $24 \cdot 5 \%(-41 \cdot 8$ to $-2 \cdot 0)$ lower incidence of stage IV disease in the MMS group (table 2). Overall, there was a 39.2\% (95\% CI 16.1 to 66.9) higher incidence of stage I or II disease and $10 \cdot 2 \%$ $(-21 \cdot 3$ to $2 \cdot 4)$ lower incidence of stage III or IV disease in the MMS group compared with the no screening group.

\begin{tabular}{|c|c|c|c|c|c|c|}
\hline & \multicolumn{5}{|l|}{ FIGO 2014 stage } & \multirow[t]{2}{*}{ Total } \\
\hline & I & II & III & IV & Unable to stage & \\
\hline \multicolumn{7}{|c|}{ Ovarian and tubal cancers (WHO 2014 classification)* } \\
\hline \multicolumn{7}{|c|}{ No screening } \\
\hline Cases & $212(20 \cdot 9 \%)$ & $73(7 \cdot 2 \%)$ & $510(50 \cdot 2 \%)$ & $208(20 \cdot 5 \%)$ & $13(1 \cdot 3 \%)$ & 1016 \\
\hline Deaths & $20(9 \cdot 4 \%)$ & $24(32 \cdot 9 \%)$ & $391(76 \cdot 7 \%)$ & $174(83 \cdot 7 \%)$ & $10(76 \cdot 9 \%)$ & $609(59 \cdot 9 \%)$ \\
\hline \multicolumn{7}{|l|}{ MMS } \\
\hline Cases & $155(29 \cdot 7 \%)$ & $42(8 \cdot 0 \%)$ & $242(46 \cdot 4 \%)$ & $78(14 \cdot 9 \%)$ & $5(1 \cdot 0 \%)$ & 522 \\
\hline Deaths & $23(14 \cdot 8 \%)$ & $16(38 \cdot 1 \%)$ & $190(78 \cdot 5 \%)$ & $62(79 \cdot 5 \%)$ & $4(80 \cdot 0 \%)$ & $291(55 \cdot 7 \%)$ \\
\hline \multicolumn{7}{|l|}{ USS } \\
\hline Cases & $121(23 \cdot 4 \%)$ & $36(7 \cdot 0 \%)$ & $253(48.9 \%)$ & $105(20 \cdot 3 \%)$ & $2(0 \cdot 4 \%)$ & 517 \\
\hline Deaths & $8(6 \cdot 6 \%)$ & $6(16 \cdot 7 \%)$ & $188(74 \cdot 3 \%)$ & $88(83 \cdot 8 \%)$ & $2(100 \cdot 0 \%)$ & $290(56 \cdot 1 \%)$ \\
\hline \multicolumn{7}{|c|}{ Between group differences in cases compared with no screening at 9.5 years after end of screening $\dagger$} \\
\hline MMS & $47 \cdot 2 \%$ (19.7 to $81 \cdot 1)$ & $15 \cdot 9 \%(-20 \cdot 7$ to $69 \cdot 4)$ & $-4 \cdot 4 \%(-18 \cdot 0$ to $11 \cdot 4)$ & $-24 \cdot 5 \%(-41 \cdot 8$ to $-2 \cdot 0)$ & .. & .. \\
\hline USS & $17 \cdot 0 \%(-6 \cdot 4$ to $46 \cdot 2)$ & $1 \cdot 1 \%(-32 \cdot 2$ to $50 \cdot 6)$ & $1 \cdot 7 \%(-12 \cdot 6$ to $18 \cdot 2)$ & $3 \cdot 4 \%(-18 \cdot 2$ to $30 \cdot 8)$ & .. &.. \\
\hline \multicolumn{7}{|c|}{ Invasive epithelial ovarian and tubal cancers (WHO 2014 classification)* } \\
\hline \multicolumn{7}{|c|}{ No screening } \\
\hline Cases & $116(12 \cdot 8 \%)$ & $69(7 \cdot 6 \%)$ & $501(55 \cdot 3 \%)$ & $208(23 \cdot 0 \%)$ & $12(1 \cdot 3 \%)$ & 906 \\
\hline Deaths & $18(15 \cdot 5 \%)$ & $24(34 \cdot 8 \%)$ & $391(78.0 \%)$ & $174(83 \cdot 7 \%)$ & $10(83 \cdot 3 \%)$ & $617(68 \cdot 1 \%)$ \\
\hline \multicolumn{7}{|l|}{ MMS } \\
\hline Cases & $91(20 \cdot 1 \%)$ & $41(9 \cdot 1 \%)$ & $237(52 \cdot 4 \%)$ & $78(17 \cdot 3 \%)$ & $5(1 \cdot 1 \%)$ & 452 \\
\hline Deaths & $22(24 \cdot 2 \%)$ & $16(39.0 \%)$ & $190(80 \cdot 2 \%)$ & $62(79 \cdot 5 \%)$ & $4(80 \cdot 0 \%)$ & $294(65.0 \%)$ \\
\hline \multicolumn{7}{|l|}{ USS } \\
\hline Cases & $55(12 \cdot 4 \%)$ & $35(7 \cdot 9 \%)$ & $249(56 \cdot 0 \%)$ & $104(23 \cdot 4 \%)$ & $2(0.4 \%)$ & 445 \\
\hline Deaths & $7(12 \cdot 7 \%)$ & $6(17 \cdot 1 \%)$ & $186(74 \cdot 7 \%)$ & $87(83 \cdot 7 \%)$ & $2(100 \cdot 0 \%)$ & $288(64 \cdot 7 \%)$ \\
\hline \multicolumn{7}{|c|}{ Between group differences in cases compared with no screening at 9.5 years after end of screening $\dagger$} \\
\hline MMS & $52 \cdot 2 \%$ (16.8 to $98 \cdot 4)$ & $15 \cdot 8 \%(-19 \cdot 4$ to $66 \cdot 4)$ & $-4 \cdot 8 \%(-18 \cdot 3$ to $10 \cdot 9)$ & $-23 \cdot 7 \%(-40 \cdot 7$ to $-1 \cdot 7)$ & .. & .. \\
\hline USS & $-8 \cdot 0 \%(-32 \cdot 7$ to $25 \cdot 7)$ & $-5 \cdot 2 \%(-35 \cdot 8$ to $39 \cdot 9)$ & $0.5 \%(-13.6$ to $16 \cdot 8)$ & $-0.8 \%(-21 \cdot 3$ to $25 \cdot 1)$ & .. &.. \\
\hline \multicolumn{7}{|c|}{$\begin{array}{l}\text { Data for cases are } \mathrm{n}(\%) \text {; data for deaths are } \mathrm{n} \text { (case fatality rate for stage); data for between group differences in cases are percentage }(95 \% \mathrm{CI}) \text {. FIGO=Federation of } \\
\text { Gynecology and Obstetrics. MMS=multimodal screening. USS=ultrasound screening. *Includes cases previously designated as primary peritoneal cancer as per WHO } 2003 \\
\text { classification. †Between group differences from a poisson model with length of analysis time as exposure variable; percentage difference taken from the incidence rate ratio, } \\
\text { where percentage difference equals incidence rate ratio minus } 1 \text { multiplied by } 100 \% \text {. }\end{array}$} \\
\hline
\end{tabular}


For the subgroup analysis of invasive epithelial ovarian and tubal cancers, the changes in stage distribution in the MMS group compared with the no screening group persisted. There was no evidence of a change in incidence in any stage in the USS group compared with the no screening group.

At censorship, $1206(0 \cdot 6 \%)$ women had died of ovarian cancer: $296(0.6 \%)$ of 50625 in the MMS group, $291(0.6 \%)$ of 50623 in the USS group, and $619(0.6 \%)$ of 101314 in the no screening group. Ovarian and tubal cancer deaths and incidence by year from randomisation

\begin{tabular}{|c|c|c|c|c|}
\hline & $\begin{array}{l}\text { Number of } \\
\text { women }(n)\end{array}$ & Deaths ( $n)$ & $\begin{array}{l}\text { Maximum } \chi^{2} \text { or mortality } \\
\text { reduction (hazard ratio) }\end{array}$ & p value \\
\hline \multicolumn{5}{|c|}{ Primary analysis* (ovarian and tubal cancer deaths) } \\
\hline MMS & 50625 & 296 & $0 \cdot 41$ & 0.58 \\
\hline USS & 50622 & 291 & $1 \cdot 23$ & 0.36 \\
\hline No screening & 101314 & 619 & .. & .. \\
\hline \multicolumn{5}{|c|}{ Subgroup outcome analysis* (invasive epithelial ovarian and tubal cancer) } \\
\hline MMS & 50625 & 295 & $0 \cdot 41$ & 0.60 \\
\hline USS & 50622 & 287 & $1 \cdot 37$ & $0 \cdot 33$ \\
\hline No screening & 101314 & 617 & .. &.. \\
\hline \multicolumn{5}{|c|}{ Secondary analysis $†$ (all data 2001-20) } \\
\hline MMS & 50625 & 296 & 0.96 (0.83 to $1.10 ; 0.068)$ & 0.52 \\
\hline USS & 50622 & 291 & $0.94(0.82$ to $1.08 ; 0.067)$ & $0 \cdot 37$ \\
\hline No screening & 101314 & 619 & $\cdot \cdot$ &.$\cdot$ \\
\hline \multicolumn{5}{|c|}{ Secondary analysis $†$ (only data 2015-20) } \\
\hline MMS & 45999 & 136 & $1.05(0.86$ to $1.30 ; 0.112)$ & 0.63 \\
\hline USS & 46079 & 128 & 0.99 (0.80 to $1.22 ; 0.107)$ & 0.91 \\
\hline No screening & 91808 & 258 & .. $\quad$ & .. \\
\hline \multicolumn{5}{|c|}{$\begin{array}{l}\text { Data are maximum } \chi^{2} \text { for primary and subgroup analyses or mortality reduction }(95 \% \mathrm{Cl} \text {; SE) for secondary analyses } \\
\text { MMS=multimodal screening. USS=ultrasound screening. }{ }^{*} \text { Versatile test. }+ \text { Cox model. }\end{array}$} \\
\hline
\end{tabular}

is detailed in the appendix (p 7). The versatile test (primary analysis) showed that there was no evidence of a reduction in ovarian and tubal cancer deaths in either the MMS $(\mathrm{p}=0 \cdot 58)$ or USS $(\mathrm{p}=0 \cdot 36)$ group compared with the no screening group (table 3 ). Figure 3 shows the Kaplan-Meier cumulative death rates, with any divergence between the screen and no screen groups being minimal. A sensitivity analysis that only considered data obtained by equivalent means of electronic health records across all three groups also showed no evidence of a difference using the versatile test result for both the MMS ( $\mathrm{p}=0 \cdot 60)$ and USS ( $=0 \cdot 37)$ group (appendix $\mathrm{p} 9)$. This analysis and other sensitivity analyses are detailed in the appendix (pp 8-9). A Cox model (secondary analysis) estimated a HR of $0.96(95 \%$ CI $0 \cdot 83-1 \cdot 10)$ for MMS versus no screening and $0 \cdot 94(0 \cdot 82-1 \cdot 08)$ for USS versus no screening. A Cox model fitted only to data from 2015 onwards (secondary analysis) estimated a HR of 1.05 (95\%CI $0.86-1 \cdot 30)$ for MMS versus no screening and $0.99(0 \cdot 80-1 \cdot 22)$ for USS versus no screening.

For the secondary outcome of invasive epithelial ovarian and tubal cancers, there were 295 (0.6\%) deaths in the MMS group, $287(0.6 \%)$ in the USS group, and $619(0 \cdot 6 \%)$ in the no screening group (table 3$)$. The cumulative death rates similarly showed no evidence of an effect of screening (appendix p 11). The versatile test for mortality reduction showed no evidence of difference in both the MMS group $(\mathrm{p}=0 \cdot 60)$ and the USS group $(\mathrm{p}=0 \cdot 33)$. The appendix shows the Royston-Parmar model fit to the non-parametric Kaplan-Meier curves (pp 12-13) and the associated hazard functions for each group (pp 14-15). All hazard functions were consistently increasing with only small differences between the screening and no screening groups. At 18 years after

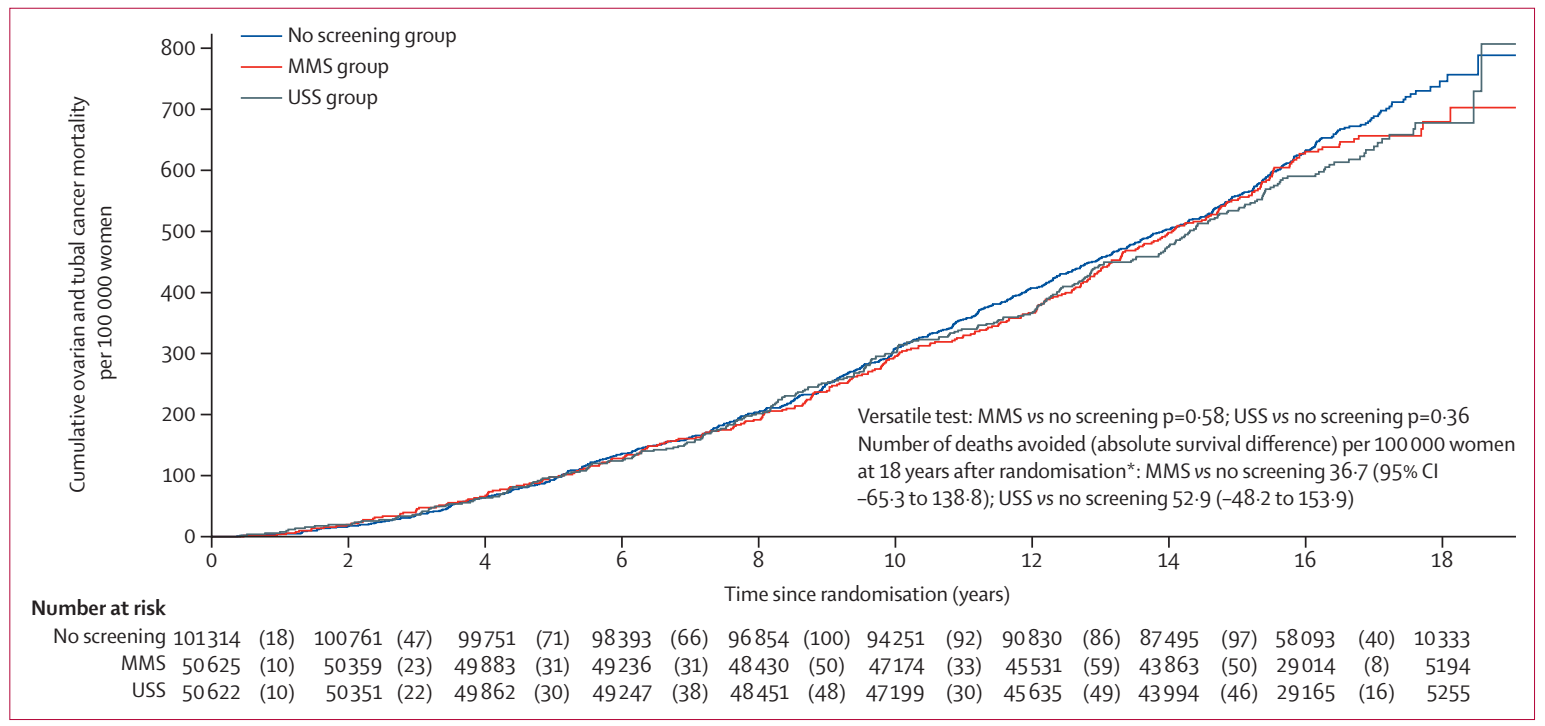

Figure 3: Kaplan-Meier cumulative mortality for ovarian and tubal cancer per 100000 women

MMS=multimodal screening. USS=ultrasound screening. *Royston-Parmar model based estimates of the effect of screening (appendix p 10). 
randomisation, the Royston-Parmar model estimates of survival differences per 100000 women were $36 \cdot 7$ (95\% CI -65.3 to $138 \cdot 8$ ) for MMS compared with no screening and $52.9(-48.2$ to $153 \cdot 9)$ for USS compared with no screening (figure 3 , appendix p 10).

Compared with no screening, in the MMS group, we observed a higher ovarian and tubal cancer case fatality rate in patients with stage I disease (20 [9.4\%] of 212 in the no screening group vs 23 [14.8\%] of 155 in the MMS group) and a lower rate with stage IV disease (174 [83.7\%] of 208 in the no screening group vs 62 [79.5\%] of 78 in the MMS group), which persisted on subgroup analysis of invasive epithelial ovarian cancer. In the USS group, the stage-specific case fatality rates appear to be similar to the no screening group, except for stage II, but numbers in this group are small (table 2).

Survival from diagnosis in women with ovarian and tubal cancer in the no screening group was better in comparison to national age and period adjusted survival rates (1 year $77 \%$ vs 68\%; 5 year $40 \%$ vs 37\%; appendix p 18).

\section{Discussion}

Our results from the largest ovarian cancer screening trial to date show that on long-term follow-up (median $16 \cdot 3$ years after randomisation), neither MMS or USS, as used in UKCTOCS, significantly reduced deaths from ovarian and tubal cancer. There was a $47.2 \%$ higher incidence of stage I cancer and $24.5 \%$ lower incidence of stage IV cancer, resulting in an overall $39.2 \%$ higher incidence of stage I or II cancer and $10.2 \%$ lower incidence of stage III or IV cancer in the MMS group than in the no screening group. General population screening for ovarian and tubal cancer with either of the screening strategies cannot be recommended based on evidence to date. The changes in stage distribution in the MMS group did not translate into mortality reduction, emphasising the importance of having disease-specific mortality as the primary outcome in ovarian cancer screening trials.

All women were treated within the NHS where, since 2004, patients are managed within a designated cancer-specific multidisciplinary team setting and ovarian cancer surgery is done only in gynaecological oncology cancer centres by subspecialty trained and accredited gynaecological oncologists. Therefore, there is unlikely to be variability in quality of care between the randomisation groups. Detailed analysis of stage and histology specific treatment is underway and will be the subject of a future report.

Achieving a mortality reduction will require a screening strategy that can detect ovarian and tubal cancer even earlier and in a larger proportion of women than we were able to achieve. Our findings make it even more important that before general population screening is introduced, any new test is shown to reduce ovarian and tubal cancer deaths in a future randomised controlled trial. These trials take many years to complete but the high compliance with annual screening in UKCTOCS suggests that women are very motivated to join them. Given that such trials take considerable time, it is probable that population screening for ovarian cancer is more than a decade away.

It is difficult to extrapolate these results to ovarian cancer screening of women at high-risk, in which the strategy has involved MMS once every 3-4 months alongside risk-reducing surgery and resulted in a significant reduction in the proportion of women diagnosed with advanced disease. ${ }^{20,21}$ Biological differences also exist between cancers in women with $B R C A$ gene mutations and the general population, which result in improved treatment responses in carriers of $B R C A$ mutations. Unfortunately, it is unlikely that the true effect of screening on mortality will ever be assessed in this population as a randomised controlled trial is challenging, and potentially very effective preventive measures such as risk reducing salpingectomy with delayed oophorectomy are being evaluated.

Our results have implications for ovarian cancer symptom awareness campaigns as most women who were screen-detected had no high-alert symptoms ${ }^{22}$ and were diagnosed earlier than would have been possible with a symptom-based approach. Our findings suggest that earlier diagnosis of invasive epithelial ovarian and tubal cancer in the symptomatic population is unlikely to translate into reduced mortality. However, it is important to note that there have been substantial advances in the treatment of advanced disease in the past 10 years since the end of screening in Dec 31, 2011. The advances in treatment, in combination with earlier diagnosis could contribute to better quality of life and improved outcomes. In addition, achieving a rapid diagnosis is of great importance to women and their families.

Our mortality results are similar to those reported for ovarian cancer screening in the Prostate Lung Colorectal Ovarian (PLCO) cancer screening trial, the only other large randomised controlled trial to report on the effects of ovarian cancer screening on mortality. ${ }^{7,8}$ In the PLCO trial, no evidence was seen of a reduction in ovarian cancer deaths between the screen and no screen arms, either at median follow-up of $12 \cdot 4^{7}$ or $14 \cdot 7^{8}$ years. However, in UKCTOCS, we found a higher incidence of stage I and lower incidence of stage IV disease in the MMS group than in the no screening group. The PLCO trial found no evidence of a difference in stage distribution between the screened and non-screened groups. ${ }^{7.8}$ The use of a longitudinal CA125 algorithm in the MMS group instead of a single CA125 cutoff, as in the PLCO trial, might have contributed to this difference. We have previously shown that a longitudinal algorithm allows us to detect disease earlier and with greater sensitivity than a single CA125 cutoff. ${ }^{23,24}$ 
For details on how to access the resources see http://uklwc. mrcctu.ucl.ac.uk/
Despite the $24.5 \%$ reduction in stage IV disease in the MMS group, the overall reduction in stage III or IV incidence 9.5 years after end of screening was only $10 \%$, with little change in stage III incidence. Previous reports have highlighted the need for a large reduction in latestage incidence as a prerequisite for reducing cancer mortality. However, it should be noted that the length of follow-up and, therefore, the dilution effect in the screen groups of inclusion of cancers diagnosed clinically after end of screening varied in these reports. An analysis of breast cancer screening trials found no reduction in breast cancer mortality in trials that achieved less than $10 \%$ reduction in stage III or IV disease and a mean reduction of $28 \%$ in trials that saw a $20 \%$ or greater reduction. ${ }^{25}$ In colorectal and lung cancer screening, much of the mortality reduction is related to reductions in stage IV disease, which has much higher mortality than earlier stages. ${ }^{26,27}$ For ovarian and tubal cancer, the high mortality associated with stage III and IV disease, combined with most women clinically detected with stage III disease, requires a substantial reduction in the incidence of both stages before a mortality reduction is possible.

There are previous instances in which increased incidence of stage I or II cancers in the screen group in screening trials did not translate to a mortality reduction. In the four early randomised controlled trials of lung cancer, ${ }^{28}$ compared with the control group, the screened groups showed significant improvements in stage distribution. However, much of the screen-detected early stage disease cases in these trials were indolent. This finding in the lung cancer trials was accompanied by a significant increase in cancer incidence in the screen groups, but no reduction in late-stage incidence. Both together suggest that overdiagnosis was the main contributor to the absence of reduction in mortality. ${ }^{28}$ This result differs from UKCTOCS, in which no significant increase in cancer incidence was observed in either of the screen groups. In the MMS group of UKCTOCS, it seems probable that the cancers shifted to an earlier stage had an intrinsic poor prognosis, which was not altered by earlier detection and the available treatments for early stage disease. Further histopathological and genetic analysis of these early stage screen-detected cancers could yield important information about the biology of ovarian cancer.

The $47.2 \%$ increase in incidence of stage I and $24.5 \%$ decrease in incidence in stage IV disease was accompanied by a higher stage I and lower stage IV case fatality rate in the MMS group. This finding persisted in subgroup analysis of invasive epithelial cancers. The findings are unlike that described previously in cancer screening trials. ${ }^{26}$ The result suggests that in the MMS group, although earlier detection of stage IV invasive epithelial ovarian and tubal cancers improved outcomes, earlier detection in stage I cancers that might have presented in later stages in the absence of screening, did not have the same effect. Stage-specific mortality and treatment will be the subject of further in-depth analyses, which will be reported in a separate publication.

The key strengths of UKCTOCS have been previously detailed $^{9}$ and include scale; multicentre design; adherence to protocol through use of a bespoke webbased trial management system with automation of key processes, remote data entry, and concurrent central monitoring; completeness of follow-up through linkage to national registries and administrative databases; and independent assignment of site and cause of death. The longitudinal algorithm we used since 2001 to interpret CA125 concentrations was innovative and forward thinking. The UK Government's Accelerating Detection of Disease Programmme includes collection of repeat biological samples that will enable building of such algorithms. We re-staged all cases using the latest FIGO 2014 criteria and revised our ovarian and tubal cancer site assignment using WHO 2014 classification to reflect current understanding of disease biology. We also changed our primary analysis approach from a constanteffect approach (proportional hazards Cox-model) to one that allows for a delayed effect (the versatile test) to reflect growing evidence that the mortality reduction in cancer-screening trials, if present, is delayed. We did this through a transparent process with publication of our methods and the expert opinions that underpinned our decision. ${ }^{18}$

Much has been learnt from the design, conduct, and analysis of UKCTOCS, which is relevant to future large-scale trials. In addition, a large bioresource of serum samples (>550000) and linked data (UKCTOCS Longitudinal Women's Cohort) has been built through the generosity of the participants. The resource includes a rare sample set of up to 11 annual blood samples from women in the MMS group. In ovarian cancer, it has allowed us to collaborate to explore new biomarkers and develop longitudinal algorithms. ${ }^{24}$ The data provides a unique opportunity to study the natural history of ovarian and tubal cancer. Important research is also underway on early detection of other cancers and risk stratification in cardiovascular disease using this bioresource.

A key limitation of the trial is the interval from end of screening in 2011 to censorship in 2020, which raises the possibility of dilution of the screening effect. However, extended follow-up after screening is the norm in screening trials and did not affect mortality reduction seen in the European Randomized Study of Screening for Prostate Cancer. ${ }^{29}$ Also, there was no variation in the ovarian cancer mortality $\mathrm{HR}$ at 15 years and 18 years from randomisation in our Royston-Parmar model (appendix p 10).

In addition, most screen-detected women were diagnosed and treated more than a decade ago, before many of the advances in clinical management (eg, widespread use of ultraradical surgery, earlier treatment modulation based on better prognostic indicators, and 
targeted therapies), which could have improved outcomes. In retrospect, second-line tests could have been further optimised so that time to diagnosis after an abnormal screen was reduced. ${ }^{23}$ Finally, clinicians could have been encouraged to intervene earlier when faced with rising biomarker concentrations and normal imaging. ${ }^{30}$ This theory is supported by modelling, which suggests that the majority of stage I, type II epithelial cancers are $0.4-1.3 \mathrm{~cm}$ in diameter and, therefore, difficult to reliably image. ${ }^{31}$

We began our mission to reduce deaths from ovarian cancer in the $1980 \mathrm{~s},{ }^{5,6}$ started our pilot randomised controlled trial in the mid-1990s, ${ }^{32}$ and then undertook UKCTOCS over the next 20 years. The journey has involved more than 200000 women who have trusted us and generously given their time, multiple UK funding agencies, numerous NHS staff both at the trial centres and more widely, and the support of many UK and international charities, and expert groups. To them, we are hugely grateful. While disappointing, the trial has provided a clear answer that our screening strategies coupled with treatment protocols available in 2001-11 (the active screening phase) did not save lives. Currently, general population screening for ovarian and tubal cancer cannot be recommended. We remain optimistic that further research will develop more effective ways to detect and treat this lethal disease. Meanwhile, the UKCTOCS biorepository with longitudinal samples provides a unique opportunity to advance early detection biomarker research.

\section{Contributors}

UM was the chief investigator from 2015 to present and was co-chief investigator from 2001-14. MP was the trial statistician. IJJ was chief investigator from 2000-14 and was a co-investigator from 2015. MP, SJS, SC, AJM, and LF were co-investigators for whole study, 2001 to present. UM, MP, IJJ, SJS, SC, AJM, LF, AG-M, MB, AR, and JKK contributed to current study concept and design. AG-M, MB, and UM did the literature search. AR, AG-M, CK, GC, JT, and SKM collected the data. Outcomes review was done by NS (chair), RM, RW, RA, AS, KW, and LC. AR and UM prepared the dataset used for the analysis. UM and AR verified the data. All statistical analysis was done by MB under the supervision of MP and independently verified by YL under the supervision of SJS. The primary analysis was also independently repeated by MR under the supervision of AJM. AR, MB, AG-M, and UM prepared the figures and tables and drafted the manuscript. UM and AR extracted the dataset. MB, SJS, YL, AJM, MR, UM, and AR had full access to the dataset. UM had final responsibility for the decision to submit for publication. All authors contributed to the interpretation of the data and revision of the manuscript. All authors approved the report before submission.

\section{Declaration of interests}

UM has stock ownership awarded by University College London (UCL) in Abcodia, which holds the licence for ROCA. She has received grants from the Medical Research Council (MRC), Cancer Research UK, the National Institute for Health Research (NIHR), and The Eve Appeal. She holds patent number EP10178345.4 for Breast Cancer Diagnostics. MP has received grants and AG-M, MB, AR, CK, GC, and SKM have been funded by grants from MRC, Cancer Research UK, NIHR, and The Eve Appeal. RM has received grants from The Eve Appeal, Rosetrees Charity, and Barts Charity, and personal fees from AstraZeneca. SJS holds the (expired) patent for ROCA, patented and owned by Massachusetts General Hospital and Queen Mary University of London, licenced to Abcodia. He reports stock options from SISCAPA Assay Technologies, and personal fees from Abcodia, Guardant Health, and
Freenome, outside the submitted work. IJJ reports grants from Eve Appeal Charity, Medical Research Council, Cancer Research UK, and NIHR during the conduct of the study. He co-invented the ROCA in 1995, it was patented by Massachusetts General Hospital and Queen Mary University of London and is owned by these universities. Massachusetts General Hospital and Queen Mary University of London granted a licence to ROCA to Abcodia in 2014. IJJ is non-executive director, shareholder, and consultant to Abcodia and has rights to royalties from sales of the ROCA. He founded (1985), was a trustee of (2012-14), and is now an Emeritus trustee (2015-present) of The Eve Appeal, one of the funding agencies for UKCTOCS. All other authors declare no competing interests.

\section{Data sharing}

The protocol is available on the study website. The individual participant data that underlie the results reported in this Article, after de-identification, will be available beginning 12 months after publication. Researchers will need to state the aims of any analyses and provide a methodologically sound proposal. Proposals should be directed to u.menon@ucl.ac.uk. Data requestors will need to sign a data access agreement and in keeping with patient consent for secondary use, obtain ethical approval for any new analyses.

\section{Acknowledgments}

We thank the volunteers without whom the trial would not have been possible and everyone involved in conduct and oversight of UKCTOCS We are very grateful to the current members of the UKCTOCS Trial Steering Committee: Henry Kitchener (Chair), Julietta Patnick, Jack Cuzick, and Annwen Jones. We are indebted to the administrative support provided by Anna Widdup, Roxanne Payne, and Jasvinder Dinza. We are indebted to the funding agencies for working together to support this long and challenging trial, and for their sustained support for UKCTOCS over many years. The long-term follow-up UKCTOCS is supported by National Institute for Health Research (NIHR HTA grant 16/46/01), Cancer Research UK, and The Eve Appeal. UKCTOCS was funded by Medical Research Council (G9901012 and G0801228), Cancer Research UK (C1479/A2884), and the UK Department of Health, with additional support from The Eve Appeal. Researchers at UCL are supported by the NIHR UCL Hospitals Biomedical Research Centre and by Medical Research Council Clinical Trials Unit at UCL core funding (MR_UU_12023). The views expressed are those of the authors and not necessarily those of the NHS, the NIHR, or the UK Department of Health and Social Care.

\section{References}

1 Cancer Research UK. Ovarian cancer survival statistics. 2018. https://www.cancerresearchuk.org/health-professional/cancerstatistics/statistics-by-cancer-type/ovarian-cancer/survival\#headingThree (accessed Jan 21, 2021).

2 Jacobs IJ, Skates SJ, MacDonald N, et al. Screening for ovarian cancer: a pilot randomised controlled trial. Lancet 1999; 353: 1207-10.

3 van Nagell JR Jr, Burgess BT, Miller RW, et al. Survival of women with type I and II epithelial ovarian cancer detected by ultrasound screening. Obstet Gynecol 2018; 132: 1091-100.

4 Kobayashi H, Yamada Y, Sado T, et al. A randomized study of screening for ovarian cancer: a multicenter study in Japan. Int J Gynecol Cancer 2008; 18: 414-20.

5 Campbell S, Bhan V, Royston P, Whitehead MI, Collins WP. Transabdominal ultrasound screening for early ovarian cancer. BMJ 1989; 299: 1363-67.

6 Jacobs I, Stabile I, Bridges J, et al. Multimodal approach to screening for ovarian cancer. Lancet 1988; 1: 268-71.

7 Buys SS, Partridge E, Black A, et al. Effect of screening on ovarian cancer mortality: the Prostate, Lung, Colorectal and Ovarian (PLCO) cancer screening randomized controlled trial. JAMA 2011; 305: 2295-303.

8 Pinsky PF, Yu K, Kramer BS, et al. Extended mortality results for ovarian cancer screening in the PLCO trial with median 15 years follow-up. Gynecol Oncol 2016; 143: 270-75.

9 Jacobs IJ, Menon U, Ryan A, et al. Ovarian cancer screening and mortality in the UK Collaborative Trial of Ovarian Cancer Screening (UKCTOCS): a randomised controlled trial. Lancet 2016; 387: 945-56.
For the protocol see http:// ukctocs.mrcctu.ucl.ac.uk/ media/1066/ukctocs-protocol_ v90_19feb2020.pdf 
10 Menon U, Gentry-Maharaj A, Ryan A, et al. Recruitment to multicentre trials-lessons from UKCTOCS: descriptive study. BMJ 2008; 337: a2079.

11 Menon U, Gentry-Maharaj A, Hallett R, et al. Sensitivity and specificity of multimodal and ultrasound screening for ovarian cancer, and stage distribution of detected cancers: results of the prevalence screen of the UK Collaborative Trial of Ovarian Cancer Screening (UKCTOCS). Lancet Oncol 2009; 10: 327-40.

12 UKCTOCS. Protocol for the United Kingdom Collaborative Trial of Ovarian Cancer Screening (UKCTOCS) and the Long Term Impact of Screening on Ovarian Cancer Mortality (LTFU UKCTOCS). 2020 http://ukctocs.mrcctu.ucl.ac.uk/media/1066/ukctocs-protocol_ v90_19feb2020.pdf (accessed Jan 1, 2021).

13 Skates SJ, Pauler DK, Jacobs IJ. Screening based on the risk of cancer calculation from bayesian hierarchical changepoint and mixture models of longitudinal markers. J Am Stat Assoc 2001; 96: 429-39.

14 Kalsi JK, Ryan A, Gentry-Maharaj A, et al. Completeness and accuracy of national cancer and death registration for outcome ascertainment in trials-an ovarian cancer exemplar. Trials 2021; 22: 88

15 Meinhold-Heerlein I, Fotopoulou C, Harter P, et al. The new WHO classification of ovarian, fallopian tube, and primary peritoneal cancer and its clinical implications. Arch Gynecol Obstet 2016; 293: 695-700.

16 Daya D, Cheung AN, Khunamornpong S. Tumors of the peritoneum: epithelial tumors of Müllerian type. In: Kurman RJ CM Herrington CS, Young RH, eds, ed. WHO classification of tumors of female reproductive organs. 4th edn. Lyon: International Agency for Research on Cancer, 2014: 92-93.

17 Tavassoli FA, Devilee P. Tumors of the breast and female genital organs. World Health Organization Classification of Tumours: Pathology and Genetics. Lyon: World Health Organization, 2003.

18 Burnell M, Gentry-Maharaj A, Skates SJ, et al. UKCTOCS update: applying insights of delayed effects in cancer screening trials to the long-term follow-up mortality analysis. Trials 2021; 22: 173.

19 Karrison TG. Versatile tests for comparing survival curves based on weighted log-rank statistics. Stata J 2016; 16: 678-90.

20 Rosenthal AN, Fraser LSM, Philpott S, et al. Evidence of stage shift in women diagnosed with ovarian cancer during phase II of the United Kingdom familial ovarian cancer screening study. J Clin Oncol 2017; 35: 1411-20.

21 Skates SJ, Greene MH, Buys SS, et al. Early detection of ovarian cancer using the risk of ovarian cancer algorithm with frequent CA125 testing in women at increased familial risk-combined results from two screening trials. Clin Cancer Res 2017; 23: 3628-37.
22 Dilley J, Allen P, Gentry-Maharaj A, et al. Symptoms reported by women with screen detected invasive epithelial ovarian cancer in the United Kingdom Collaborative Trial of Ovarian Cancer Screening (UKCTOCS). Int J Gynecol Cancer 2015; 25 (suppl 2): 423-24.

23 Menon U, Ryan A, Kalsi J, et al. Risk algorithm using serial biomarker measurements doubles the number of screen-detected cancers compared with a single-threshold rule in the United Kingdom Collaborative Trial of Ovarian Cancer Screening. J Clin Oncol 2015; 33: 2062-71.

24 Blyuss O, Burnell M, Ryan A, et al. Comparison of longitudinal CA125 algorithms as a first-line screen for ovarian cancer in the general population. Clin Cancer Res 2018; 24: 4726-33.

25 Tabár L, Yen AM, Wu WY, et al. Insights from the breast cancer screening trials: how screening affects the natural history of breast cancer and implications for evaluating service screening programs. Breast J 2015; 21: 13-20.

26 Schoen RE, Pinsky PF, Weissfeld JL, et al. Colorectal-cancer incidence and mortality with screening flexible sigmoidoscopy. N Engl J Med 2012; 366: 2345-57.

27 de Koning HJ, van der Aalst CM, de Jong PA, et al. Reduced lung cancer mortality with volume CT screening in a randomized trial. N Engl J Med 2020; 382: 503-13.

28 Strauss GM, Gleason RE, Sugarbaker DJ. Chest X-ray screening improves outcome in lung cancer. A reappraisal of randomized trials on lung cancer screening. Chest 1995; 107 (suppl): 270-79S.

29 Schröder FH, Hugosson J, Roobol MJ, et al. Prostate-cancer mortality at 11 years of follow-up. N Engl J Med 2012; 366: 981-90.

30 Menon U, Gentry-Maharaj A, Ryan A, et al. Serial CA125 interpreted using the risk of ovarian cancer algorithm can detect ovarian cancer in absence of ultrasound abnormalities Innovations \& Progress in Healthcare for Women, 2nd International Meeting: Prevention, Screening and Risk Prediction in Women's Health; London; Oct 16, 2009. AB.021 16 (abstr).

31 Brown PO, Palmer C. The preclinical natural history of serous ovarian cancer: defining the target for early detection. PLoS Med 2009; 6: e1000114.

32 Menon U, Skates SJ, Lewis S, et al. Prospective study using the risk of ovarian cancer algorithm to screen for ovarian cancer. J Clin Oncol 2005; 23: 7919-26. 